\title{
Multiplicative functions with difference tending to zero
}

\author{
by \\ Eduard Wirsing (Ulm) and Don Zagier (Bonn)
}

1. Introduction. Denote by $\Delta$ and $Q$ the operators on arithmetic functions defined by

$$
\Delta f(n):=f(n+1)-f(n), \quad Q f(n):=f(n+1) / f(n) .
$$

There are two objectives to the present note. The first is related to a result of the first named author, published jointly with Tao Yuan-Sheng and Shao Pin-Tsung [3]:

THEOREM 1. If an additive function $f: \mathbb{N} \rightarrow \mathbb{R} / \mathbb{Z}$ has the property $\Delta f(n) \rightarrow 0$ as $n \rightarrow \infty$, then

$$
f(n)=c \log n+\mathbb{Z} \quad \text { with some constant } c \in \mathbb{R} .
$$

We will show that the proof of this theorem as given there, although already quite short, can still be shortened and made clearer by a certain rearrangement of the main arguments.

Theorem 1 has an obvious translation to the multiplicative setting: If a multiplicative function $f: \mathbb{N} \rightarrow \mathbb{C}$ with modulus 1 has the property $Q f(n) \rightarrow 1$, then it is of the form $n^{s}$ with $s$ a purely imaginary complex number. In fact this statement remains true even if we drop the condition of unimodularity, except that now of course the exponent can be arbitrary:

Theorem 2. If $f: \mathbb{N} \rightarrow \mathbb{C}$ is multiplicative and $Q f(n) \rightarrow 1$ as $n \rightarrow \infty$, then $f(n)=n^{s}$ with some $s \in \mathbb{C}$.

On the other hand, for $f: \mathbb{N} \rightarrow \mathbb{C}$ of modulus 1 the conditions " $Q f(n) \rightarrow 1$ " and " $\Delta f(n) \rightarrow 0$ " are equivalent, so a different strengthening of Theorem 1 is given by the following result, which is our second main objective:

Theorem 3. If $f: \mathbb{N} \rightarrow \mathbb{C}$ is multiplicative and $\Delta f(n) \rightarrow 0$, then either $f(n)=n^{s}$ with $s \in \mathbb{C}, 0 \leq \operatorname{Re} s<1$, or else $f(n) \rightarrow 0$ as $n \rightarrow \infty$.

2000 Mathematics Subject Classification: Primary 11A25. 
Theorem 3, which confirms an older conjecture of Kátai, was stated as a consequence of Theorem 1 by the first named author in a letter to Kátai in 1984, and is quoted and applied as "a result of Wirsing from 1984" in a paper of Kátai and Phong from 1996 [1]. Unfortunately it was never published. We shall supply here a proof of Theorem 3 via Theorem 2 .

The results of this paper extend - and use - a well known theorem of Erdős on additive functions, of which we append a short proof for the reader's convenience.

2. Proof of Theorem 1 . We denote by $\|\cdot\|$ the norm in $\mathbb{R} / \mathbb{Z}$, defined by $\|\kappa x\|=\left|x-x^{\prime}\right|$, where $\kappa$ is the canonical mapping from $\mathbb{R}$ to $\mathbb{R} / \mathbb{Z}$ and $x^{\prime}$ the integer nearest to $x$.

I. There is a function $F: \mathbb{N} \rightarrow \mathbb{R}$ such that $\kappa \circ F=f$ and $|\Delta F(n)|=$ $\|\Delta f(n)\|$. Just choose each $F(n+1)$ from $(F(n)-1 / 2, F(n)+1 / 2]$. Consequently, $\Delta F(n) \rightarrow 0$.

II. In terms of $F$ the additivity of $f$ is expressed by stating that

$$
\gamma(a, b):=F(a b)-F(a)-F(b) \text { is in } \mathbb{Z} \text { if }(a, b)=1 .
$$

III. For given $a$ and bounded gaps between $n, n^{\prime}$ we have $\gamma\left(a, n^{\prime}\right)-$ $\gamma(a, n)=F\left(a n^{\prime}\right)-F(a n)-F\left(n^{\prime}\right)+F(n)=o(1)$. Thus the subsequence of the (integral!) $\gamma(a, n)$ with $(a, n)=1$ stabilizes to some integer $\delta(a)$, and the whole sequence converges, i.e.

$$
\lim _{n \in \mathbb{N}} \gamma(a, n)=\delta(a) \in \mathbb{Z} .
$$

IV. Consider the easily checked identity:

$$
\gamma(a, b)=\gamma(a, b c)+\gamma(b, c)-\gamma(a b, c) .
$$

If we send $c$ to $\infty$, then in view of III we obtain

$$
\gamma(a, b)=\delta(a)+\delta(b)-\delta(a b),
$$

and here $b \rightarrow \infty$ yields $\delta(a b)-\delta(b) \rightarrow 0$, that is,

$$
\delta(a b)=\delta(b) \quad \text { for all } b \geq n_{a}, n_{a} \text { suitable. }
$$

The first of these relations is best expressed if we introduce the new function

$$
G(n):=F(n)+\delta(n) .
$$

Then (1) states that $G$ is completely additive.

V. Let us look at (2). In particular it implies that if $b \geq n_{2}$ then $\delta(b)=$ $\delta(2 b)$. Now also $2 b \geq n_{2}$ etc., hence $\delta(2 b)=\delta(4 b)$ etc., $\delta(b)=\delta\left(2^{k} b\right)$ for all $k$. Furthermore for all large $k$ (as soon as $2^{k} \geq n_{b}$ ) another application 
of (2) gives $\delta(b)=\delta\left(2^{k}\right)$. Since this is independent of $b$ we have:

The function $\delta$ is constant from some point $\left(=n_{2}\right)$ on.

VI. From this and I we obtain $\Delta G(n) \rightarrow 0$ for the (completely) additive function $G$. Then by Erdös's Theorem (cf. $\S 4$ ) it follows that $G(n)=c \log n$ for some constant $c \in \mathbb{R}$ and finally, since $\delta(n) \in \mathbb{Z}$ and $f=\kappa \circ F=\kappa \circ G$,

$$
f(n)=c \log n+\mathbb{Z}
$$

\section{Proof of Theorems 2 and 3}

Proof of Theorem 2. If we write $f(n)=|f(n)| e^{2 \pi i g(n)}$ then under the given assumptions $\log |f|: \mathbb{N} \rightarrow \mathbb{R}$ and $g=(2 \pi)^{-1}$ arg $f: \mathbb{N} \rightarrow \mathbb{R} / \mathbb{Z}$ are additive and $\Delta \log |f(n)|=\log |Q f(n)| \rightarrow \log 1=0, \Delta g(n)=(2 \pi)^{-1} \arg Q f(n)$ $\rightarrow(2 \pi)^{-1} \arg 1=0$, so $\log |f(n)|=\sigma \log n$ and $g(n)=\tau(2 \pi)^{-1} \log n+\mathbb{Z}$ by Erdös's Theorem and Theorem 1 respectively. Thus, as claimed, $f(n)=$ $n^{\sigma+i \tau}$.

Proof of Theorem 3. Note that $\Delta f(n) \rightarrow 0$ implies $Q f(n) \rightarrow 1$, provided $|f(n)|$ is bounded below by some positive constant $\mu$ :

$$
|Q f(n)-1|=\left|\frac{\Delta f(n)}{f(n)}\right| \leq \frac{|\Delta f(n)|}{\mu} .
$$

So in this case Theorem 2 applies and gives $f(n)=n^{s}$. Obviously $\operatorname{Re} s<1$, since $\Delta f(n) \rightarrow 0$ implies $f(n)=o(n)$.

It remains to show $f(n) \rightarrow 0$ if $f$ is not bounded in this way. In fact a weaker assumption suffices and Theorem 3 will follow immediately from

LEMmA. If $f: \mathbb{N} \rightarrow \mathbb{C}$ is multiplicative, $\Delta f(n) \rightarrow 0$, and there is an $a \in \mathbb{N}$ such that $|f(a)|<1$, then $f(n) \rightarrow 0$ as $n \rightarrow \infty$.

Proof. Let $a$ be a fixed number such that $|f(a)|=: q<1$. By assumption, we have $|\Delta f(n)| \leq \varepsilon / a^{2}$ for $n \geq n_{0}(\varepsilon)$. To each $n \in \mathbb{N}$ we attach a sequence $n, n^{\prime}, n^{\prime \prime}, \ldots, n^{(k)}$ by the modified division algorithm $n^{(i-1)}=a n^{(i)}+r_{i}$ that allows $0 \leq r_{i}<a^{2}$ but requires that $a$ and $n^{(i)}$ be coprime. We stop at the first index $k$ for which $n^{(k)}<n_{0}(\varepsilon)+a^{2}$. Breaking the gaps up into $r_{i}$ steps of length 1 we see $\left|f\left(n^{(i-1)}\right)-f(a) f\left(n^{(i)}\right)\right|<\varepsilon$ for $i=1, \ldots, k$. Thus

$$
\left|f\left(n^{(i-1)}\right)\right|<q\left|f\left(n^{(i)}\right)\right|+\varepsilon \text {. }
$$

The total result from these inequalities is

$$
\begin{aligned}
|f(n)| & <\varepsilon+q\left(\varepsilon+q\left(\varepsilon+\ldots+q\left(\varepsilon+q\left|f\left(n^{(k)}\right)\right|\right) \ldots\right)\right) \\
& <\varepsilon(1-q)^{-1}+q^{k}\left|f\left(n^{(k)}\right)\right| .
\end{aligned}
$$

Since $\varepsilon$ is arbitrary, $f\left(n^{(k)}\right)$ stays bounded once $\varepsilon$ is fixed, and $k$ tends to infinity as $n$ does, we see that indeed $f(n) \rightarrow 0$. 


\section{Appendix: Erdös's Theorem}

THEOREM 4 (Erdős, 1946). If an additive function $f: \mathbb{N} \rightarrow \mathbb{R}$ has the property $\Delta f(n) \rightarrow 0$ as $n \rightarrow \infty$, then

$$
f(n)=c \log n \quad \text { with some constant } c \in \mathbb{R} .
$$

The following proof is taken from [2]. For convenience, assume first that $f$ is completely additive, which is all that is needed for our application. Choose an integer $g \geq 2$ and attach to each $n \in \mathbb{N}$ a sequence $n, n^{\prime}, n^{\prime \prime}, \ldots, n^{(k)}$ by the $g$-adic division algorithm $n^{(i-1)}=g n^{(i)}+r_{i}$ where $0 \leq r_{i}<g$, which terminates when $n^{(k)}<g$. Breaking the gaps up into steps of length 1 we see $\left|f\left(n^{(i-1)}\right)-f\left(n^{(i)}\right)-f(g)\right|<\varepsilon$, for any $\varepsilon$, as long as $n^{(i)}$ is sufficiently large, and bounded for the remaining $i$. The total result from these inequalities is

$$
|f(n)-k f(g)|<k \varepsilon+O_{\varepsilon}(1),
$$

in other words,

$$
f(n)=k f(g)+o(k) .
$$

Since $k \sim \log n / \log g$ as $n \rightarrow \infty$ we have

$$
f(n)=\frac{\log n}{\log g} f(g)+o(\log n)
$$

The very first impression is disappointment: An asymptotic relation rather than the expected identity? But the asymptotic behavior is independent of the choice of $g$. Therefore $f(g) / \log g$ is a constant! The same proof works with restricted additivity if one uses the modified division algorithm as in the lemma.

\section{References}

[1] I. Kátai and B. M. Phong, On some pairs of multiplicative functions correlated by an equation, in: New Trends in Probability and Statistics, Vol. 4 (Palanga, 1996), VSP, Utrecht, 1997, 191-203.

[2] E. Wirsing, Additive and completely additive functions with restricted growth, in: H. Halberstam and C. Hooley (eds.), Recent Progress in Analytic Number Theory, II (Durham, 1979), Academic Press, 1981, 231-280.

[3] E. Wirsing, Y. S. Tao and P. T. Shao, On a conjecture of Kátai for additive functions, J. Number Theory 56 (1996), 391-395.

Universität Ulm

Helmholtzstraße 18

D-89069 Ulm, Germany

E-mail: wirsing@mathematik.uni-ulm.de
Max-Planck-Institut für Mathematik

Vivatsgasse 7

D-53111 Bonn, Germany E-mail: zagier@mpim-bonn.mpg.de 